\title{
Adenoma thyroid with supraclavicular tuberculous lymphadenitis Mas- querading as malignancy thyroid with Metastasis - Diagnostic dilemma
}

\section{Nagendra Mahendra ${ }^{1}$ Abhijeet Ingle ${ }^{2}$ Dharmendra kumar borad ${ }^{3}$ Majed Abdul Basit Momin2}

1. Consultant ENT, Head \& Neck surgeon, Department of Ear, Nose \& Throat. Yashoda Hospital. Malakpet, Nalgonda x-roads, Hyderabad- 500036.

2. Consultant pathologist ,Department of Laboratory Medicine , Yashoda Hospital Malakpet, Nalgonda x-roads, Hyderabad-500036.

3. Consultant radiologist, Yashoda Hospital, Malakpet, Nalgonda x-roads, Hyderabad - 500036.

DOI : 10.47799/pimr.0802.22

Abstract: Thyroid swelling or goitre is one of the commonest presentations among female patients more than males attending ENT out patients. The clinical importance of thyroid swelling is related to evaluating their functional status and assess, whether it is benign,malignant, hypo or hyper active.Even if benign should look for pressure symptoms which justifies surgery.Tuberculous lymphadenitis is the most common form of extrapulmonary tuberculosis. Coexistence of thyroid swelling with supraclavicular lymph node mimicking malignancy with metastatic deposit is unusual and always to be evaluated. We report a case of a 45 -year female,who on outside initial evaluation was diagnosed as follicular carcinoma of thyroid. However clinical examination revealed a palpable supraclavicular lymph node. Thus subjected to a repeat FNA (fine needle aspiration) from thyroid which revealed benign thyroid follicular adenoma and FNA from supraclavicular lymph node revealed tuberculous lymphadenitis, which was further confirmed by histological sections post operatively.

This case emphasizes the need for thorough clinical examination and utility of image guided FNA to rule out the biological nature of these lesions.

Key features: Thyroid adenoma; Extrapulmonary tuberculosis; FNAC; Histology

\section{Introduction}

Thyroid nodule is a discrete lesion in thyroid gland, that is radiologically distinct from the surrounding thyroid parenchyma ${ }^{[1]}$. Thyroid nodules commonly encountered in females and its percentage of prevalence in the general population varies depending on mode of discovery as 2-6\% by palpation, $20-35 \%$ by ultrasound and $8-65 \%$ as per autopsy data $^{[2]}$. Though thyroid nodules are common, their clinical significance is mainly related to excluding malignancy ( 4.0 to $6.5 \%$ of all thyroid nodules), evaluating their functional status and if they cause any pressure symptoms ${ }^{[3]}$.

Tuberculous lymphadenitis is the most common form of extrapulmonary tuberculosis. The left supraclavicular lymph node (Virchow's node) may be involved by metastatic malignancies, including those of abdominal or pelvic origin ${ }^{[4]}$. Fine needle aspiration cytology (FNAC) plays a vital role as a rapid diagnostic technique because of its simplicity, costeffectiveness, early availability of results, accuracy, and minimal invasion ${ }^{[5]}$. In our case during a clinicalexamination found a left supraclavicular lymph node. Repeat FNA thyroid in our center revealed thyroid adenoma and supraclavicular lymph node showed tuberculous lymphadenitis.

\section{Case Report:}

A 45 year, old woman presented with swelling in front of neck for four months.There was no complain of pain,change in voice, difficulty in swallowing or breathing. There was a history of mild cough on and off. General physical examination revealed swelling over neck, moves on swallowing, non-tender, firm and no sign of inflammation(Figure 1, pink arrow). Nose and throat examination was normal. There were palpable left supraclavicular lymph node, of size $1.5 \times 1.5 \mathrm{~cm}$, mobile, deep seated and non tender (Figure 1, blue arrow).

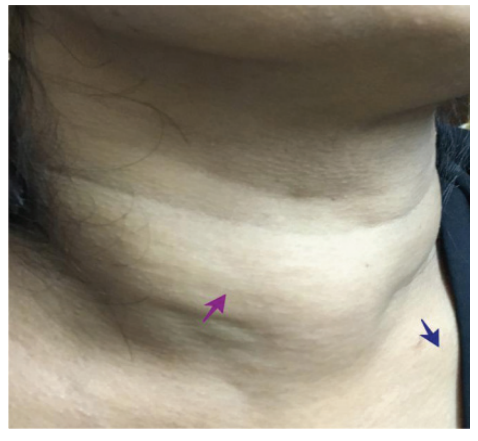

Figure:1 Right thyroid lobe nodule (pink arrow) and left supraclavicular swelling (blue arrow)

Outside investigations revealed normal hemogram and biochemical values. Thyroid profile was within normal range. Ultrasound neck confirmed solid thyroid nodule in right lobe of thyroid, measuring $60 \times 50 \times 36 \mathrm{~mm}$ and nodes in left supraclavicular area suggestive of metastatic neck node. HRCT neck performed(Coronal and axial plane) which shows, well defined, heterogeneous right thyroid lobe nodule with central necrosis and few specks of calcification peripherally,measuring 65×52×35 mm (figure 2).Thyroid nodule compressing and displacing the trachea towards left. Inferiorly it extended into the retrosternal region.Also left supraclavicular lymph node noted with loss of fatty hilum (Figure 2). Overall features suggestive of neoplastic etiology. 


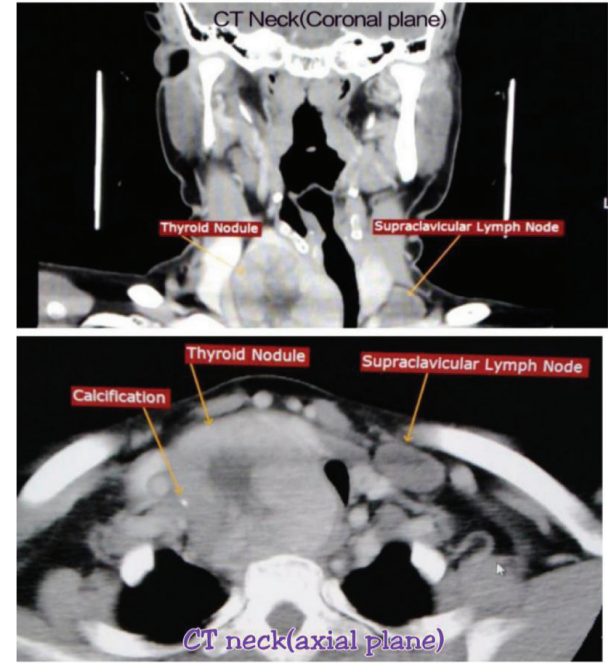

Figure: 2 HRCT coronal and axial plane show right thyroid lobe nodule with peripheral calcification and central necrosis. Left supraclavicular lymph node with loss of fatty hilum

Preoperative FNA was performed from both primary thyroid mass and left supraclavicular lymph nodes.

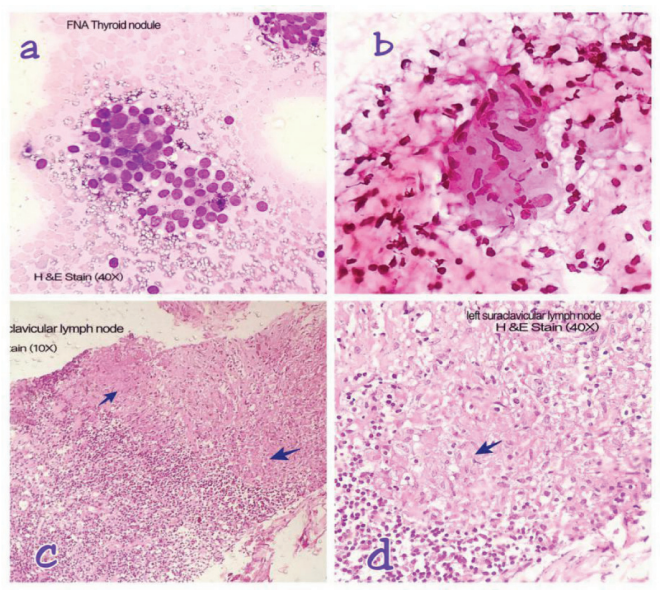

Figure 3a: FNAC Thyroid smear show thyroid follicular cells

Figure 3b: FNAC left supraclavicular lymph node showing granulomas composed of epithelioid cells.

Figure3c: Lymph node histology $(10 \mathrm{X})$ showing granulomas

Figure3d: Lymph node histology $(40 \mathrm{X})$ showing granulomas with epithelioid cells surrounded by lymphocytes.

Cytological examination of thyroid nodule FNA(Figure 3a) shows follicular epithelial cells arranged in sheets and follicles with round regular nuclei and evenly distributed chromatin suggestive of benign follicular adenoma. Left supraclavicular lymph node cytology showed (Figure 3b) granulomas composed of epithelioid cells surrounded by lymphocytes. Areas of caseation necrosis seen. Acid Fast Bacilli came positive on special staining (Ziehl-Neelson). Excision biopsy performed from the left supraclavicular lymph node(Figure3c\&3d).
Histological and molecular Gene Xpert findings confirmed tuberculous lymphadenitis. Anti tuberculus treatment initiated and after three months, elective Right hemithyroidectomy was performed.

On macroscopic examination, the thyroid nodule was well encapsulated, measuring $7 \times 4.5 \times 4.5 \mathrm{~cm}$ (Figure 4 small inlet box).

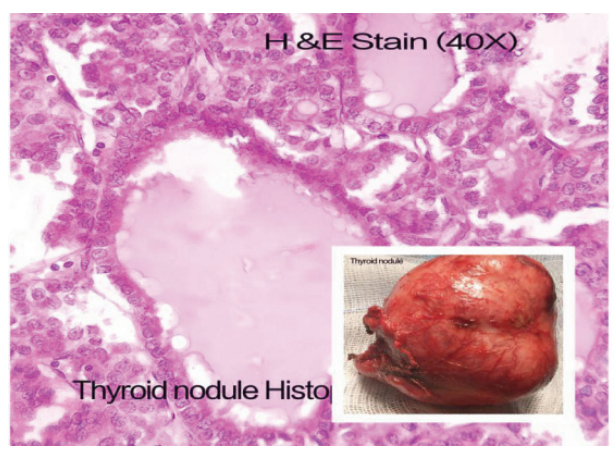

Figure4: Histology of thyroid nodule with gross excised nodule(inlet box)

Microscopic examination of histological sections showed thyroid follicles of varying sizes, with thin colloid with no evidence of malignancy. Post operative period was uneventful and discharged after three days with continuation of antituberculous drugs.

\section{Discussion:}

Thyroid nodules are common. They are noticed by patient or family members or either on self palpation by the patient.At times detected during physical examination by the clinician or incidentally during a radiological procedure. Thyroid nodules can be caused by both benign and malignant conditions. Benign conditions includes colloid nodule, simple or hemorrhagic cyst, follicular adenoma, Hashimotos thyroiditis or subacute thyroiditis[1]. Malignant conditions are seen 4.0 to $6.5 \%$ in thyroid nodules and these are papillary cancer, follicular cancer, medullary carcinoma, oncocytic carcinomas, anaplastic and thyroid lymphoma ${ }^{[3]}$.

The enlarged supra-clavicular lymphadenopathy can be the first clinical sign of underlying malignancy, especially in elderly. However, many benign conditions such as reactive hyperplasia and infectious etiology related to enlarged lymph nodes ${ }^{[6]}$. FNAC with sampling for gen $x$ pert testing is an important tool for early diagnosis. Gene X-pert testing with rifampicin resistance test in aspirated samples has shown significantly higher levels of sensitivity and specificity for diagnosis of EPTB as compared to cytomorphology and Z-N staining microscopy alone $^{[7]}$.

The coexistence of thyroid carcinomas and cervical tuberculous lymphadenitis has been described in several case reports from Asian and European journals. In a case series of Papillary thyroid carcinomas patients with cervical lymphadenopathy, 
$72 \%$ were eventually diagnosed with tuberculous lymphadenitis after an initial consideration of metastatic from thyroid carcinoma ${ }^{[8]}$. In another case series by chang et al, 1693 patients with papillary thyroid carcinomas and lymphadenopathy, 28 had TB lymphadenitis of these, seven also had concomitant nodal metastasis from papillary thyroid carcinoma $^{[9]}$.

FNAC (fine needle aspiration cytology) is the gold standard test for evaluating thyroid nodules, particularly, under ultrasound guidance for representative aspirate and to lower the chances of false negative results compared to procedure done without guidance. It is safe, economical and accurate way for evaluating thyroid nodules ${ }^{[4]}$. The cytological examination results reported using various classification, of which The Bethesda System for Thyroid Cytopathology is most commonly used. The cytological findings are crucial in guiding the further steps in management of nodules ${ }^{[10]}$. In our case patient came with FNA suggestive of follicular neoplasm with extensive lymphadenopathy on radiological workup suggestive of metastatic spread. Although ultrasound is a reasonably sensitive diagnostic method to detect lesions but difficult to ascertain the benign from neoplastic lesions. Reevaluation of Thyroid nodule and supraclavicular FNA suggestive of benign follicular thyroid adenoma with supraclavicular tuberculous Iymphadenitis. Further histological examination of supraclavicular lymph node confirm tuberculous lymphadenitis.

Surgical removal performed for benign nodules if they are causing pressure or structural symptoms. TSH suppressive therapy has no role in the management of benign nodules. For malignant lesions total thyroidectomy or near-total thyroidectomy with lymph node dissection is performed ${ }^{[1]}$. In retrospect, prompt TB workup in our patient identified the disease preoperatively leading to earlier initiation of antituberculous therapy and sparing the patient an unnecessary neck dissection.

To conclude, supraclavicular lymphadenopathy in a patient with thyroid swelling is not always suggestive of metastatic spread. Tuberculosis should be considered an important differential in the etiology of supraclavicular lymphadenopathy in a patient with thyroid swelling. Fine needle aspiration (FNA) is an accurate and cost effective way to evaluate thyroid nodules and lymphadenopathy. Thorough preoperative evaluation helps accurate diagnosis, management and to avoid morbidity related to unnecessary surgical procedure.

\section{REFERENCES}

1. Tamhane and Gharib; Thyroid nodule update on diagnosis and management ;Clinical Diabetes and Endocrinology (2016) 2:17

2 Dean DS, Gharib H. Epidemiology of thyroid nodules. Best Pract Res Clin Endocrinol Metab. 2008;22(6):901-11.
3. Lin JD, Chao TC, Huang BY, Chen ST, Chang HY, Hsueh C. Thyroid cancer in the thyroid nodules evaluated by ultrasonography and fine-needle aspiration cytology. Thyroid. 2005;15(7):708-17.

4. Cervin JR, Silverman JF, Loggie BW, Geisinger KR. Virchow's node revisited. Analysis with clinicopathologic correlation of 152 fine-needle aspiration biopsies of supraclavicular lymph nodes. Arch Pathol Lab Med 1995;119:727-30.

5. Nasuti JF, Mehrotra R, Gupta PK. Diagnostic value of fineneedle aspiration in supraclavicular lymphadenopathy: A study of 106 patients and review of literature. Diagn Cytopathol 2001;25:351-5

6. Rathod KM, Shah SA. A study of metastatic lesion of lymph node by fine needle aspiration cytology. Natl J Community Med 2012;3:708-10.

7. Automated Real time Nucleic acid Amplification Technology for rapid and simultaneous Detection of Tuberculosis and rifampicin Resistance:Xpert MTB/RIF System; Policy statement ; WHO, 2011.

8. M. Iqbal, A. Subhan, and A. Aslam, "Papillary thyroid carcinoma with tuberculous cervical lymphadenopathy mimicking metastasis," Journal of the College of Physicians and Surgeons Pakistan, vol. 21, no. 4, pp. 207-209, 2011

9. S. M. Kim, H. H. Jun, H. J. Chang et al., "Tuberculosis cervical lymphadenopathy mimics lateral neck metastasis from papillary thyroid carcinoma," ANZ Journal of Surgery, vol. 86, no. 6, pp. 495-498, 2016.

10. S. Z. Ali and E. S. Cibas, Eds., The Bethesda System for Reporting Thyroid Cytopathology. Definitions, Criteria and Explanatory Notes, Springer, New York, NY, USA, 2010.

How to cite this article: Nagendra Mahendra, Ingle A, Dharmendra kumar borad, Majed Abdul Basit Momin . Adenoma thyroid with supraclavicular tuberculous lymphadenitis Masquerading as malignancy thyroid with Metastasis - Diagnostic dilemma. Perspectives in Medical Research 2020; 8 (2):106-108. DOI : 10.47799/pimr.0802.22 Sources of Support: Nil, Conflict of interest: None declared 